\title{
Direct Laser Fabrication of Cylindrical Lenses in Wide Band Gap Materials
}

\author{
Sebastian Buettner*, Michael Pfeifer, and Steffen Weissmantel \\ Laserinstitut Hochschule Mittweida, 09648, Mittweida, Germany
}

\begin{abstract}
The Fluorine laser microstructuring technique enables the direct laser fabrication of cylindrical lenses and lens arrays thereof in wide band gap materials. For the mask projection technique, a special mask geometry was calculated, which allows the fabrication of cylindrical lenses with a nearly optimum curved surface. Based on our results of processing fused silica and borosilicate glass, we investigated the possibility to apply these microstructuring technique to wide band gap materials like calcium fluoride. The radius of curvature (ROC) can be adjusted by the process parameters laser pulse fluence and pulse-to-pulse overlap in a range of 130 to $450 \mu \mathrm{m}$ micrometer. A minimal surface roughness of $100 \mathrm{~nm}$ RMS can be reached.
\end{abstract}

\section{Introduction}

Micro optics are becoming more and more important to deal with technical problems. A wide range of techniques were developed to manufacture optical elements in the micrometer range in the last few years and in particular for the fabrication of cylindrical lenses and lens arrays [1, 2]. One of these is the fluorine laser microstructuring. This technique enables the direct laser fabrication of micro lenses in glass and especially in wide band gap materials like calcium fluoride. This is achieved by the short wavelength of $157 \mathrm{~nm}$ and the corresponding high photon energy of $7.9 \mathrm{eV}$ of the fluorine laser. The microstructuring is done by a fully automated highprecision laser micromachining station. The pulse duration of the laser is $25 \mathrm{~ns}$, the maximum repetition rate is $200 \mathrm{~Hz}$ and the maximum pulse energy is $30 \mathrm{~mJ}$.

Based on our research on the microstructuring of blaze gratings [3], diffractive optical elements (DOEs) [4], and Fresnel lenses [5] we further developed these technique for the fabrication of cylindrical lenses with a nearly optimal shaped lens surface and a defined ROC. In a first step we fabricated cylindrical lenses and arrays thereof in fused silica and borosilicate glass [6]. In combination with the $\mathrm{CO}_{2}$ laser smoothing as a follow up treatment process, we can fabricate these kind of optical elements with a surface roughness in a range of a few nanometers. In a next step we applied this technique to wide band gap materials.

\section{Results and Discussion}

Because of the short wavelength of the fluorine laser the microstructuring of wide band gap materials is possible. However, even materials with a higher band gap than the photon energy can be processed, as can be seen in Fig. 1 .
The fabrication of cylindrical lenses requires the knowledge of the specific material parameters ablation threshold $\mathrm{H}_{\mathrm{th}}$ and absorption coefficient $\alpha$. These parameters can be calculated using the Lambert-Beer's law and the ablation depth per pulse depending on the laser pulse fluence. Therefore, a parameter study for every used material has been done. In Fig. 1 the ablation depth per pulse depending on the laser pulse fluence is shown for different wide band gap materials.

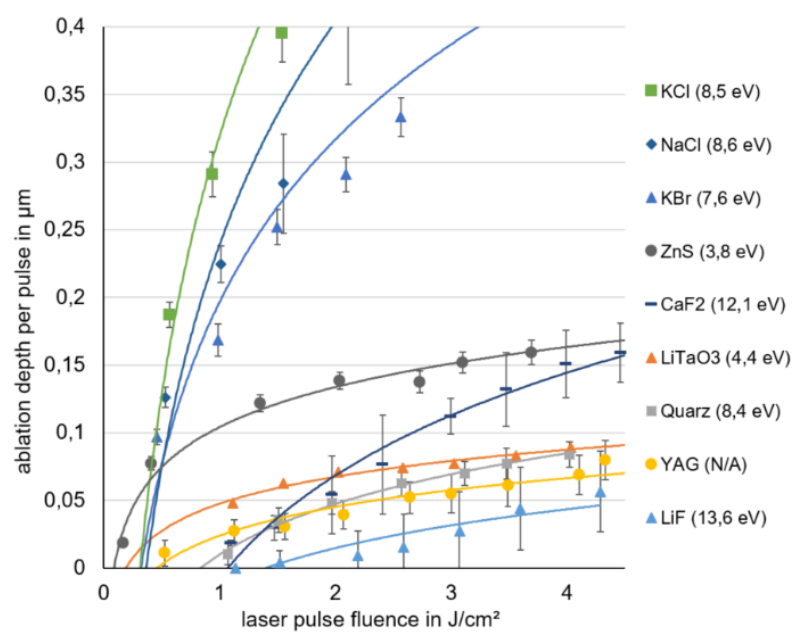

Fig. 1. Ablation depth per pulse for different wide band gap materials.

For the demonstration of the fabrication of cylindrical lenses in wide band gap materials, we choose calcium fluoride as material for our investigations. The determined ablation threshold of this material is $\mathrm{H}_{\mathrm{th}}=1.07 \mathrm{~J} / \mathrm{cm}^{2}$ and the absorption coefficient is $\alpha=7.861 \cdot 10^{4} \mathrm{~cm}^{-1}$. Furthermore, we used the mask, which we calculated for previous investigations on fused silica and borosilicate glass. A micrograph of this mask is shown in Fig. 2.

* Corresponding author: buettner@hs-mittweida.de 


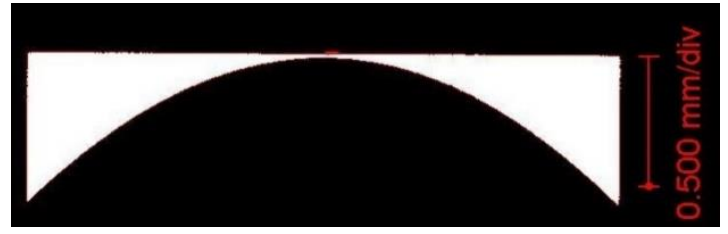

Fig. 2. Optical micrograph of the calculated mask.

For the fabrication of cylindrical lenses in calcium fluoride, the laser pulse fluence was varied in a range of 2 to $4.5 \mathrm{~J} / \mathrm{cm}^{2}$ and the pulse-to-pulse overlap in a range of 95 to $99 \%$. In general, the ROC of the lenses depending on the laser pulse fluence and the pulse-to-pulse overlap. The pulse-to-pulse overlap is defined by the relation of the maximum mask aperture to the pulse-to-pulse distance. For this material, the fabrication of nearly optimal shaped lenses requires a defined laser pulse fluence of $3.5 \mathrm{~J} / \mathrm{cm}^{2}$. At this laser pulse fluence, the course of the profile section is in a good agreement with the best fit ROC, as can be seen in Fig. 3. The ROC of these lenses are within the range of 130 to $450 \mu \mathrm{m}$. Furthermore, the surface roughness of these lenses is in a range of $100 \mathrm{~nm}$ RMS.

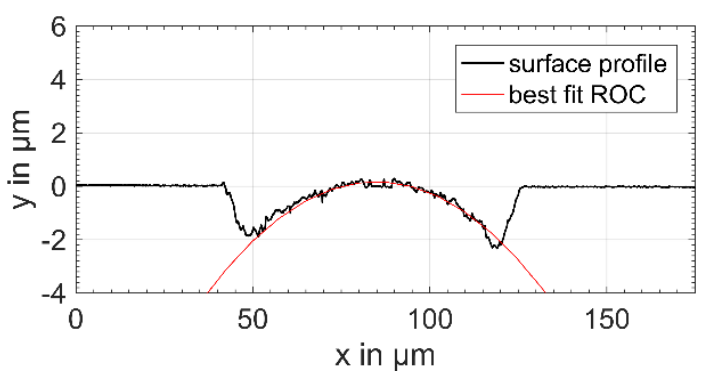

Fig. 3. Profile section of a cylindrical lens fabricated in calcium fluoride $\left(\mathrm{H}=3.5 \mathrm{~J} / \mathrm{cm}^{2}\right.$, overlap $\left.98 \%\right)$.

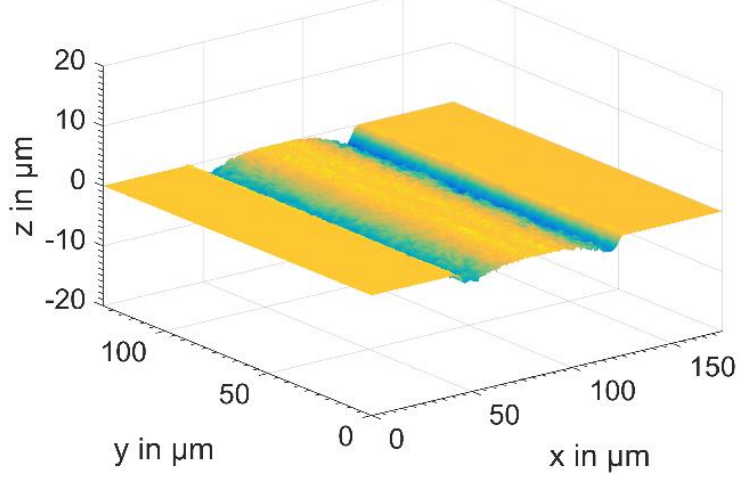

Fig. 4. Three dimensional micrograph of a cylindrical lens fabricated in calcium fluoride $\left(\mathrm{H}=3.5 \mathrm{~J} / \mathrm{cm}^{2}\right.$, overlap $\left.98 \%\right)$.

For other laser pulse fluences, the profile section deviates from the circular form. This is caused by a strong increase of the surface roughness at higher and an inconvenient ablation behavior at lower laser pulse fluences. The reason for the latter is the high ablation threshold, which lead to an incomplete removal of the material at the vertex of the lenses. This problem can be solved by adapting the mask geometry in the region of the vertex. However, the waveoptical simulation show a focussing effect of the lenses. For this, the measured three dimensional surface data were used to calculate the phase shift and the electrical field strength of a propagating electromagnetic wave. Due to the roughness of the surface, a part of the light is scattered, as can be seen in Fig 5.

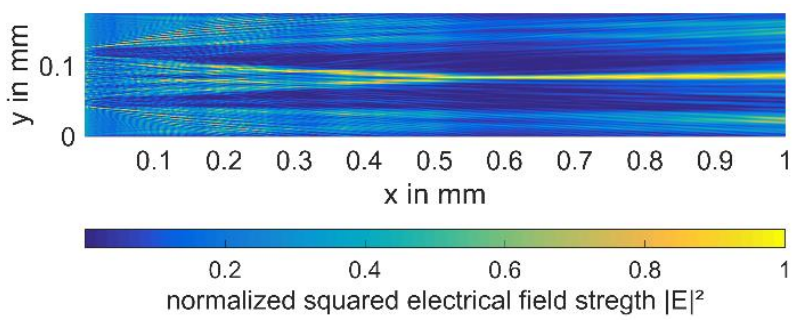

Fig. 5. Squared electrical field strength $|E|^{2}$ normalized to 1 depending on the propagation direction $\mathrm{x}$, calculated from a profile section of a cylindrical lens fabricated in calcium fluoride $(\lambda=532 \mathrm{~nm}, \mathrm{n}=1.43)$.

\section{Conclusion}

The fabrication of cylindrical lenses in calcium fluoride can be done using the fluorine laser microstructuring technique. At a laser pulse fluence of $3.5 \mathrm{~J} / \mathrm{cm}^{2}$, lenses with a nearly optimal curved surface can be fabricated, with an ROC in the range from 130 up to $450 \mu \mathrm{m}$. In general, the ROC depending on the pulse-to-pulse overlap. For lower and higher laser pulse fluences, the surface profile section deviates from the circular form. In particular at lower fluences, the microstructuring process is affected by the incomplete removal of the material. Regarding to this, the calculation of an adapted mask geometry can enlarge the field of applicable parameters. This may enables the fabrication of lenses at a lower laser pulse fluence, which can lead to a reduction of the surface roughness. Furthermore, we want to use an ion beam for follow-up treatment. In this way, we can reach a further improvement of the lens surface.

\section{Acknowledgements}

The authors gratefully acknowledge the financial support of the present work by the European Union and the Free State of Saxony.
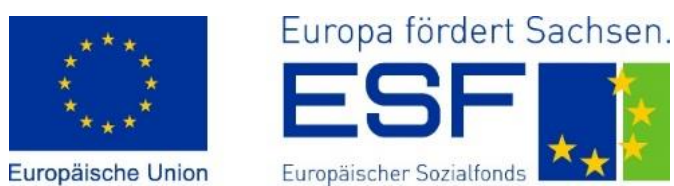

\section{References}

[1] Z. Luo, K. Yin, X. Dong, J. Duan, Opt. Mat. 78 (2018)

[2] H.-K. Choi, M.S. Ahsan, D. Yoo, I.-B. Sohn, Y.-C. Noh, J.T. Kim, H.-M. Kang, Opt. Laser Technol. 75 (2015)

[3] M. Pfeifer, S. Weissmantel, G. Reisse, Appl. Phys. A 112, 1 (2013)

[4] M. Pfeifer, F. Jahn, A. Kratsch, B. Steiger, S. Weissmantel, Proc. of PHOTOPTICS (2014)

[5] M. Pfeifer, S. Büttner, R. Zhang, M. Serbay, S. Weißmantel, In Sci. Rep. of Univ. of Appl. Sci. Mittweida, 2 (2017)

[6] S. Buettner, M. Pfeifer, S. Weissmantel, Proc. of PHOTOPTICS (2019) 